\title{
Complementary study on the electrical and structural properties of poly(3-alkylthiophene) and its copolymers synthesized on ITO by electrochemical impedance and Raman spectroscopy
}

\author{
Danielly Cristina Bento - Elaine Cristina Rodrigues Maia • \\ Thiago Nogueira Marques Cervantes • Clarissa de Almeida Olivati • \\ Guy Louarn · Henrique de Santana
}

Received: 22 July 2014 / Accepted: 6 October 2014/Published online: 11 October 2014

(C) Springer Science+Business Media New York 2014

\begin{abstract}
Targeting the use of organic conjugated polymers in organic solar cells, this study looks at the electrochemical properties of poly(3-alkylthiophene) films: poly(3-methylthiophene), poly(3-hexylthiophene), poly(3octylthiophene) and its copolymers electrochemically synthesized on a tin-doped indium oxide (ITO) substrate. Electrochemical impedance spectroscopy (EIS) was used to monitor the change in the electrochemical behavior of these films on the ITO and the charge transfer resistance $\left(\mathrm{R}_{\mathrm{CT}}\right)$ values were determined in open circuit potential (OCP) and at different overpotentials. Together with the EIS, the ex situ and in situ Raman spectroscopy was used to characterize the influence of aromatic radical cation and dication species, present in the polymer matrix of homo and copolymers, in the management processes seen in the Nyquist and Bode-phase diagrams for different systems obtained on ITO. The EIS results in OCP showed an decrease in resistance demonstrating increased conductivity with the copolymerization, and through the Bode-phase diagram and the ex situ Raman spectra, these changes were related to the oscillation of the radical cation and dication along the polymer matrix. By studying Nyquist and Bodephase diagrams in the overpotentials, an increase in $R_{C T}$
\end{abstract}

D. C. Bento - E. C. R. Maia - T. N. M. Cervantes ·

H. de Santana $(\bowtie)$

Departamento de Química - CCE, Universidade Estadual de Londrina, Londrina, PR 86051-990, Brazil

e-mail: hensan@uel.br

C. de Almeida Olivati

Faculdade de Ciência e Tecnologia (FCT), Unesp,

Presidente Prudente, SP 19060-900, Brazil

G. Louarn

Institut des Matériaux Jean Rouxel, Université de Nantes-CNRS,

44322 Nantes, France values in higher potentials was seen that could be related to the bipolaronic process, which after the deconvolution of the in situ Raman spectra, was possible to relate these results to the stabilization of the dication species in the homo and copolymers matrix when subjected to high potential.

\section{Introduction}

Among the conducting polymers, poly(3-alkylthiophene) (P3ATs) exhibits good physical and chemical stability, and since they are $\pi$-conjugated systems, they can undergo oxidation when electrochemically neutral, forming $p$-type doped systems of electron donors [1]. This doping generates charge carriers such as a radical cation (polaron) and dication (bipolaron) along the polymer chain, which are electrostatically compensated by inserting anions from the electrolyte solution [2-6]. Due to its structural properties, the electrochemical processes involved in conductivity of P3ATs are very important to understand this system aimed at using it in organic solar cells [2,7-10]. Some applications of P3ATs use a tin oxide doped with indium (ITO) as a substrate, making a clear and colorless conductive film on a glass [7, 11, 12]. The ITO presents appropriate work function value to organic materials, i.e. it has a good ability to collect electrons generated by electron donors, allowing this property to vary according to changes in its active surface [13-17].

To study the electrochemical properties of modified polymeric films, the electrochemical impedance spectroscopy (EIS) has been an important tool in understanding electronic transport in semiconductor systems, such as double layer capacitance, impedance of diffusion, charge transfer velocity, charge transfer processes and solution 
resistance, that is, processes that must be present for photovoltaic cells to operate. Some studies have been developed using EIS at different overpotentials and open circuit potential (OCP) with the help of cyclic voltammetry (CV) to determine the charge transfer on films of poly(3-methylthiophene) (P3MT) and poly(3-octylthiophene) (P3OT) and its copolymers with poly diphenylamine (PDFA) synthesized in similar conditions on platinum. Variation in charge transfer resistance as the overpotential increase was seen in the Nyquist diagrams, and the different electrochemical behavior in OCP due to the structural changes that incurred in the polymer chain $[4,18,19]$.

The electrochemical behavior of P3ATs and their copolymers synthesized in different electrolyte solutions on platinum, it was also investigated in overpotential that showed a strong dependence on doping with a nature to support electrolytes, such as $\mathrm{LiClO}_{4}, \mathrm{Et}_{4} \mathrm{NBF}_{4}$ and $\mathrm{TBAPF}_{6}$, relating to transporting the charge inside the mobile polymer, entry and exit of the dopant counter ions, favored by stabilizing the radical cation and dication species in the polymer matrix [3, 20-22]. Marchesi et al. [23] using CV and EIS techniques to understand the electrochemical degradation processes found, the decrease in the redox capacity of polymers with an increase in the number of voltammetric cycles and with the data from the Nyquist graph obtained by EIS, related to redox capacity variation with mobile ions along the polymer chain. Benedict et al. [18] determined the variation in the resistivity values $\left(\mathrm{R}_{\mathrm{CT}}\right)$ and double layer capacitance $\left(\mathrm{C}_{\mathrm{DL}}\right)$ of P3MT and P3OT and their copolymers with the PDFA through the EIS in OCP and overpotential, and with Bode-phase diagram related to the two constants of the phase where the charge transfer processes occurred involving the radical and dication cation species.

The data obtained by EIS has also contributed to the study of the time constants using the Bode diagram. The Bode-phase has helped to identify the charge transfer processes, in which the process at low frequencies may be associated with polaronic conduction, which represents the delocalization movement of positive charges. The process at high frequencies has been associated with electronic conduction, which represents the movement of electrons along the conduction band of the polymer. Thus, the conductivity of ions and electrons at the polymer/electrode interface and polymer/solution interface can be directly related to the behavior of the time constants seen in the Bode-phase diagram [22].

This work aims to synthesize the P3MT, P3HT and P3OT homopolymers and copolymers on ITO electrode aiming to improve the diffusion/migration mechanisms in these materials, compared with the behavior that these films previously generated on platinum electrode. So, to monitor the variation in electrochemical behavior of these
Table 1 Experimental conditions for synthesis by chronoamperometry (CA) of the films on ITO

\begin{tabular}{lllllll}
\hline Films & \multicolumn{2}{l}{$\begin{array}{l}\text { Concentration } \\
\left(\mathrm{mol} \mathrm{L}^{-1}\right)\end{array}$} & & $\begin{array}{l}\text { Time } \\
(\mathrm{s})\end{array}$ & $\begin{array}{l}\mathrm{E}(\mathrm{vs} . \mathrm{Ag} / \\
\mathrm{AgCl})\end{array}$ & $\begin{array}{l}\mathrm{j} \\
\left(\mathrm{mA} \mathrm{cm}^{-2}\right)\end{array}$ \\
\cline { 2 - 4 } & 3MT & $3 \mathrm{HT}$ & $3 \mathrm{OT}$ & & & \\
\hline P3MT & 0.035 & - & - & 240 & 1.70 & 3.97 \\
P3HT & - & 0.040 & - & 240 & 1.75 & 3.90 \\
P3OT & - & - & 0.040 & 240 & 1.75 & 4.00 \\
CP3(MT- & 0.035 & 0.040 & - & 240 & 1.70 & 5.33 \\
HT) & & & & & & \\
CP3(MT- & 0.035 & - & 0.040 & 240 & 1.70 & 8.67 \\
$\begin{array}{c}\text { OT) } \\
\text { CP3(HT- }\end{array}$ & - & 0.040 & 0.040 & 240 & 1.75 & 8.00 \\
OT) & & & & & & \\
\hline
\end{tabular}

films on ITO, the $\mathrm{R}_{\mathrm{CT}}$ values in OCP were determined by EIS, and the $R_{C T}$ values at different overpotentials were determined by the EIS with the help of CV. Together with the EIS, the in situ and ex situ Raman spectroscopy was used to understand the influence of aromatic species, radical cations and dications present in the polymer matrix of homo and copolymers, in the charge transfer processes seen in the Nyquist and Bode-phase diagrams of the different systems obtained on ITO.

\section{Experimental}

\subsection{Reagents}

The monomers, 3-methylthiophene $\left(\mathrm{C}_{5} \mathrm{H}_{7} \mathrm{~S}\right)$, 3-hexylthiophene $\left(\mathrm{C}_{10} \mathrm{H}_{16} \mathrm{~S}\right)$ and 3-octylthiophene $\left(\mathrm{C}_{12} \mathrm{H}_{20} \mathrm{~S}\right)$ were supplied by Aldrich and used as received, lithium perchlorate $\left(\mathrm{LiClO}_{4}\right)$ was used as a supporting electrolyte, supplied by Acros Organics, with $99 \%$ purity. Acetonitrile $\left(\mathrm{CH}_{3} \mathrm{CN}\right)$ was obtained from JT Baker with $99.5 \%$, purity, HPLC grade.

\subsection{Synthesis by chronoamperometry (CA) and study by cyclic voltammetry (CV)}

A standard three-electrode cell was used in all electrochemical experiments. The homo and copolymer films were synthesized on ITO, with average area $0.30 \mathrm{~cm}^{2}$. All potentials were measured with reference to $\mathrm{Ag} / \mathrm{AgCl}$ deposited in a Luggin capillary in a solution of $0.100 \mathrm{~mol} \mathrm{~L}^{-1}$ of $\mathrm{LiClO}_{4}$ in acetonitrile $\left(\mathrm{LiClO}_{4} / \mathrm{ACN}\right)$. A platinum plate $(10 \times 10 \times 0.50 \mathrm{~mm})$ was used as the auxiliary electrode.

The conditions described in Table 1 were used for the electrochemical synthesis of P3MT, P3HT and P3OT films 
Table 2 Values of maximum frequency $(f)$, of open circuit potential (OCP) and the overpotential $(\eta)$ for homo and copolymers

\begin{tabular}{lrll}
\hline Films & $f(\mathrm{~Hz})$ & $\begin{array}{l}\mathrm{OCP}(\mathrm{E} \mathrm{vs.} \\
\left.\mathrm{Ag}_{(\mathrm{s})} / \mathrm{AgCl}_{(\mathrm{s})}\right)\end{array}$ & $\begin{array}{l}\eta\left(\mathrm{E} \mathrm{vs.} \mathrm{Ag}_{(\mathrm{s})} /\right. \\
\left.\mathrm{AgCl}_{(\mathrm{s})}\right)\end{array}$ \\
\hline P3MT & 31,623 & 0.248 & $1.10 ; 1.50 ; 1.80$ \\
P3HT & 7,943 & 0.039 & $0.90 ; 1.05 ; 1.80$ \\
P3OT & 15,849 & 0.678 & $0.70 ; 0.90 ; 1.90$ \\
CP3(MT-HT) & 12,589 & 0.272 & $1.00 ; 1.10 ; 2.00$ \\
CP3(MT-OT) & 15,849 & 0.028 & $1.00 ; 1.50 ; 2.00$ \\
CP3(HT-OT) & 12,589 & 0.719 & $1.00 ; 1.20 ; 2.00$ \\
\hline
\end{tabular}

and their copolymers with each other. The latter ones were obtained from a solution of 1:1 mixture of monomers as obtained and named by Cervantes et al. [3].

The CA and CV curves were obtained with potentiostat/ galvanostat equipment, model Autolab PGSTAT 302N, linked to a microcomputer with NOVA 1.8 software.

\subsection{Electrochemical impedance spectroscopy (EIS)}

The impedance diagrams carried out on the open circuit potential (OCP) and overpotentials $(\eta)$, were obtained using the potentiostat Autolab PGSTAT $302 \mathrm{~N}$ with a FRAM32 impedance module, varying the frequency of $100 \mathrm{kHz}$ to $0.01 \mathrm{~Hz}$.

Table 2 shows the maximum frequency (f) values, OCP and $\eta$ for each system used in the EIS.

\subsection{Spectroscopic characterization}

The ex situ and in situ Raman spectra were obtained using a portable DeltaNu Advantage $532{ }^{\circledR}$ Raman Spectrometer, excited at $532 \mathrm{~nm}$ with a resolution of $8 \mathrm{~cm}^{-1}$. DeltaNu Nuspec software was used, baseline resources were used to remove background fluorescence. While obtaining in situ Raman spectra, the same potential for $\eta$ was applied, Table 2, with the help of a Microquimica potentiostat device, model MQPG-01, linked to the microcomputer. The potentials were applied with reference to $\mathrm{Ag} / \mathrm{AgCl}$ deposited on a Luggin capillary in a solution of $0.100 \mathrm{~mol}$ $\mathrm{L}^{-1}$ of $\mathrm{LiClO}_{4} / \mathrm{ACN}$.

\section{Results and discussion}

The Nyquist diagrams, $-Z^{\prime \prime}$ versus $Z^{\prime}$ curves, for the homo and copolymers on ITO in $0.100 \mathrm{~mol} \mathrm{~L}^{-1}$ of $\mathrm{LiClO}_{4} / \mathrm{ACN}$ obtained by EIS in OCP are shown in Fig. 1.

In Fig. 1, a semicircle was seen in most graphs, with the exception of P3OT and CP3HT-OT, which had two semicircles. In previous work on these P3ATs and its copolymers, synthesized on platinum, only a semicircle was found $[3,18]$. The emergence of the two semicircles in the diagrams of the P3OT and CP3HT-OT films synthesized on ITO, is due to the charge transfer process between the ITO/polymer and polymer/solution interfaces [3, 24]. The effect that occurs in the first semicircle, recorded at higher frequencies, may be related to the characteristics of interfacial processes in terms of resistance in the ITO/ polymer interface, characteristics not found on these synthesized films on platinum [3].

For the Nyquist diagrams for copolymers CP3MT-HT, CP3MT-OT and CP3HT-OT obtained with OCP, the semicircles reduced on the polymer/solution interface, resulting in a lower charge transfer resistance $\left(\mathrm{R}_{\mathrm{CT}}\right)$ compared to homopolymer semicircles. This decrease in the $\mathrm{R}_{\mathrm{CT}}$ with the copolymerization was also seen between the P3ATs and the PDFA [18] and between P3ATs [3], both synthesized on platinum.

$\mathrm{R}_{\mathrm{CT}}$ values determined by IES for P3MT, P3HT and P3OT were $1.60 \times 10^{2}, 1.81 \times 10^{3}$ and $1.34 \times 10^{3} \Omega \mathrm{cm}^{2}$ and for CP3MT-HT, CP3MT-OT and CP3HT-OT copolymers $1.15 \times 10^{2}, 66.24$ and $4.57 \times 10^{2} \Omega \mathrm{cm}^{2}$, respectively. These values show an decrease in resistance demonstrating increased conductivity of the copolymers relative to their respective homopolymers. The resistivity sequence for the copolymers is given by CP3(HT-OT) $>$ CP3(MT-HT) $>$ CP3(MT-OT) and P3HT $>$ P3OT $>$ P3MT for the homopolymers.

These results demonstrate that the copolymerization favored the formation of new materials with different electrochemical behavior than their respective homopolymers. As seen for the films synthesized on platinum [3, 18, 25], the resistivity sequence between the homopolymer films on ITO also showed a decrease in the resistive behavior with the side chain of the polymer matrix, that is, a P3MT for having the smallest side chain among P3ATs with the lowest resistivity. Thus, it was found that resistive behavior of homopolymers in an open circuit potential behavior was dependent on the side chain in the polymer matrix.

It was found that the synthesized films on ITO showed semicircles with smaller diameters, i.e. a smaller $\mathrm{R}_{\mathrm{CT}}$ when compared to that synthesized on platinum [3, 18]. The values seen demonstrate that the synthesis on ITO results in films that are resistant up to $10^{4} \Omega \mathrm{cm}^{2}$ times less than on platinum; that gave an $\mathrm{R}_{\mathrm{CT}}$ of $3.73 \times 10^{5}, 2.28 \times 10^{7}$, $1.35 \times 10^{7}, 3.43 \times 10^{5} \Omega \mathrm{cm}^{2}$ for P3MT, P3HT, P3OT and CP3MT-HT, respectively.

In order to help interpret the findings by the IES technique in OCP, Raman spectra were obtained aimed at monitoring the segments present in the polymeric material that justifies the change in resistive behavior seen between homo and copolymers. For better viewing of the results, the 

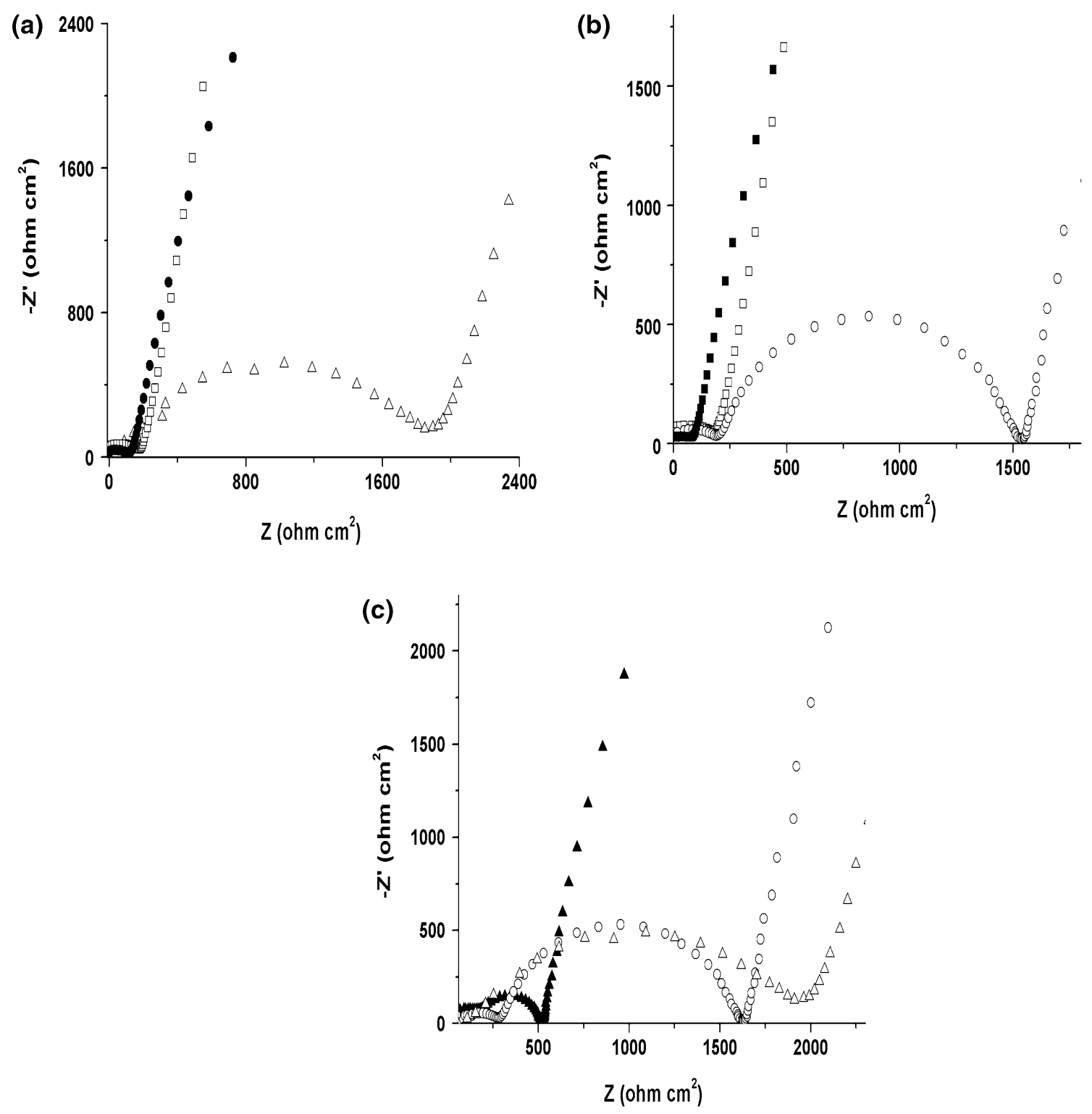

Fig. 1 Nyquist diagrams obtained in OCP for the following films deposited on ITO in $0.100 \mathrm{~mol} \mathrm{~L}^{-1}$ of $\mathrm{LiClO}_{4} / \mathrm{ACN}$ : a P3MT (opened square), P3HT (opened triangle) and CP3MT-HT (filled

spectral region between 1,400 and $1,580 \mathrm{~cm}^{-1}$ of the Raman spectra was deconvoluted, because this region refers to the characteristic frequencies of symmetric $\mathrm{C}=\mathrm{C}$ stretching of the thiophenic ring, sensitive to the changes that it sustained in the thiophenic ring of the homopolymers [6, 26-28]. Figure 2 shows the homo and copolymer deconvoluted ex situ Raman spectra generated on ITO in $0.100 \mathrm{~mol} \mathrm{~L}^{-1}$ of $\mathrm{LiClO}_{4} / \mathrm{ACN}$.

In ex situ deconvoluted Raman spectra, a set of frequencies can mostly be seen with three characteristic structures to radical cation, dication and aromatic species. In the ex situ spectra, Fig. 2, bands were seen at 1,428-1,438, $1,443-1,461$ and $1,460-1,483 \mathrm{~cm}^{-1}$ attributed to the circle); b P3MT (opened square); P3OT (opened circle) and CP3MTOT (filled square); c P3HT (filled triangle); P3OT (opened circle) and CP3HT-OT (filled triangle)

aromatic, c species, respectively [6, 26-28] with variation in the relative intensity for different samples.

In the ex situ spectrum of homopolymers, Fig. $2 a-c$ the band relating to the aromatic species had become intense. This behavior due to the presence of the resonance Raman effect for this species, which absorbs close to the excitation wavelength of the Raman spectrum. Since the band attributed to the radical cation species in $1,460 \mathrm{~cm}^{-1}$ had become intense in the ex situ spectra for P3HT and P3OT,

Fig. 2 Deconvoluted ex situ Raman spectra: a P3MT, b P3HT, c P3OT, d CP3MT-HT, e CP3MT-OT, f CP3HT-OT synthesized on the ITO in $0.100 \mathrm{~mol} \mathrm{~L}^{-1}$ of $\mathrm{LiClO}_{4} / \mathrm{ACN}$ 

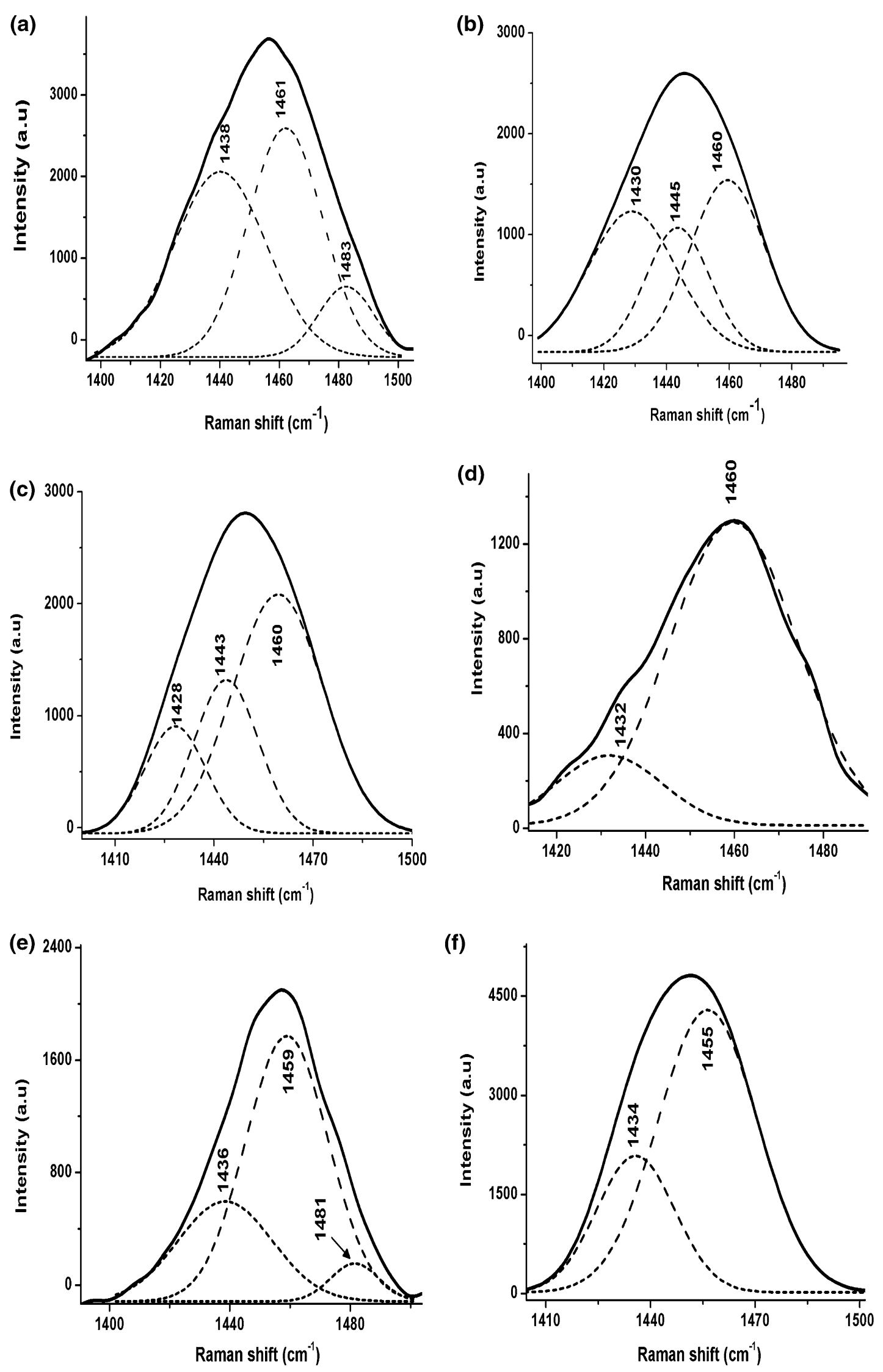
Fig. 2 b, c. In the ex situ spectra for the CP3MT-HT, CP3MT-OT and CP3HT-OT copolymers, Fig. $2 \mathrm{~d}-\mathrm{f}$, the band attributed to the radical cation species on the P3HT and P3OT homopolymers were more intense. The band at $1,481 \mathrm{~cm}^{-1}$ was even seen in the CP3MT-OT spectrum, a characteristic of the radical cation segments present in the P3MT spectrum.

Given the results of the ex situ Raman spectra deconvolution, the less resistive behavior of the copolymers, compared to homopolymers, seen in Nyquist diagrams in OCP, Fig. 1, can be related to better stabilization of the radical cation in the matrix of these materials. This smaller
$\mathrm{R}_{\mathrm{CT}}$ may have been caused by an increase in the "diffusion-migration" process of $\mathrm{ClO}_{4}{ }^{-}$doping due to increased interaction between the positively charged segments and this counter-ion, which contributes to an decrease in resistance demonstrating increased conductivity along the copolymer chain, and the largest $\mathrm{R}_{\mathrm{CT}}$ may be related to the low accumulation of $\mathrm{ClO}_{4}{ }^{-}$caused by the absence of the radical cation segment in this copolymer matrix [3].

These results demonstrate the stabilization variation of these three species in different synthesized films on ITO, and that this variation may be influencing the resistive behavior of homo and copolymers. (a)

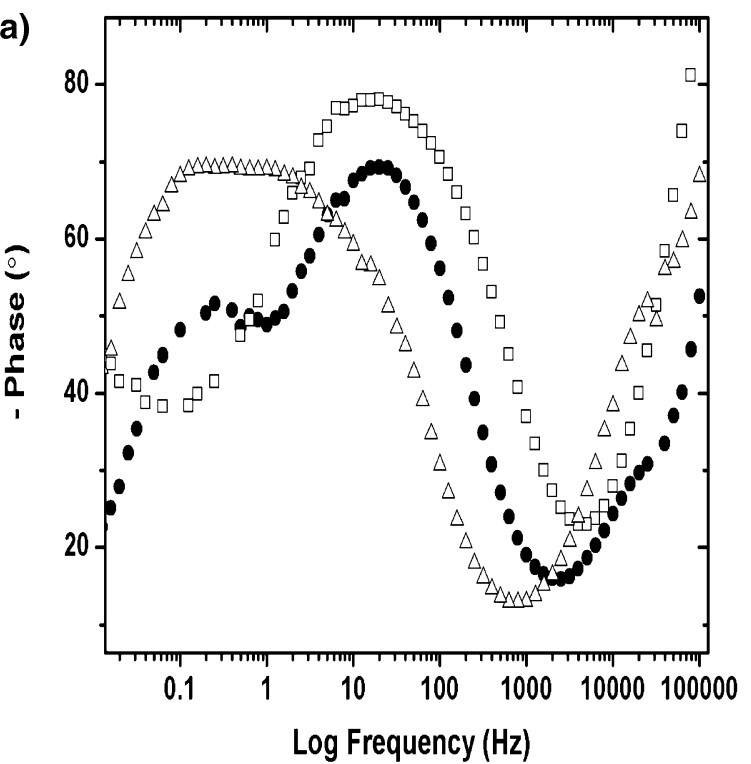

(b)

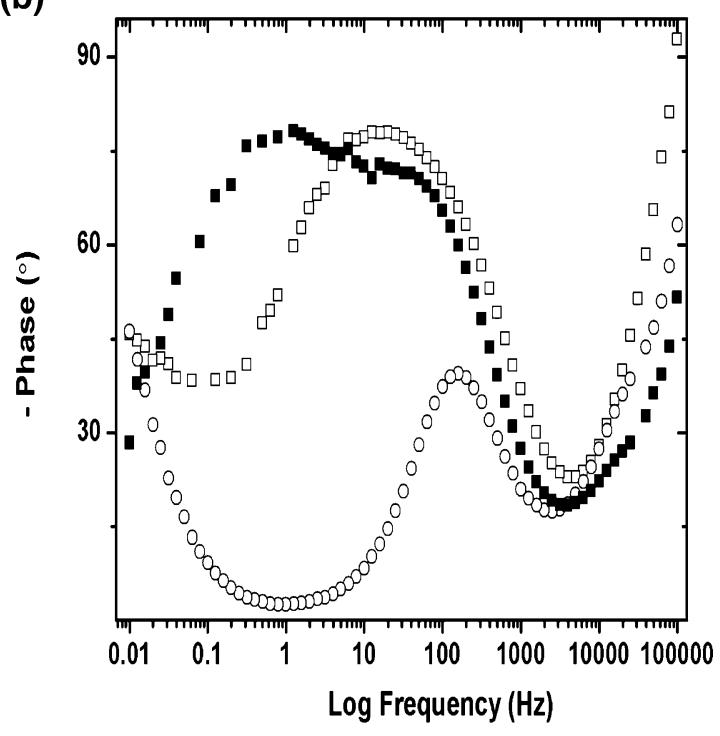

(c)

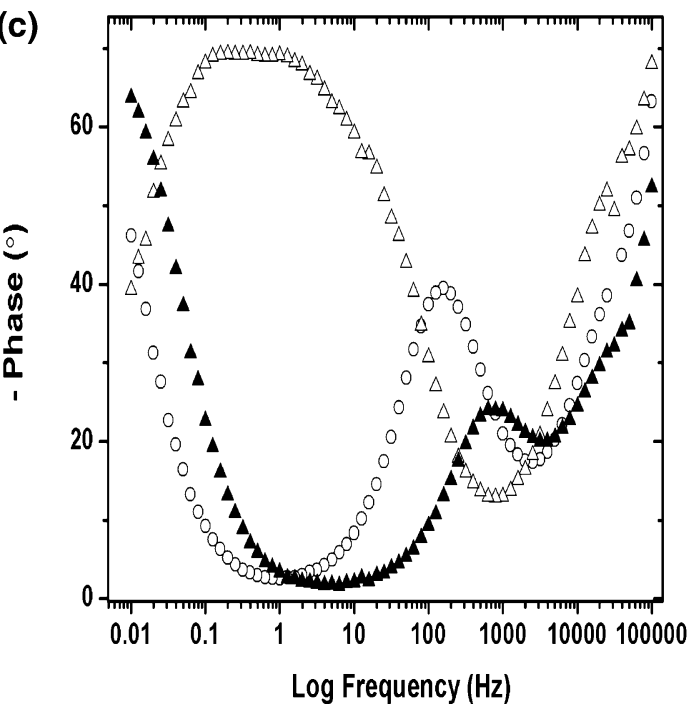

Fig. 3 Bode-phase diagrams generated in OCP for the following films deposited on ITO in $0.100 \mathrm{~mol} \mathrm{~L}^{-1}$ of $\mathrm{LiClO}_{4} / \mathrm{ACN}$ : a P3MT (opened square), P3HT (opened triangle) and CP3MT-HT (filled circle), b P3MT (opened square), P3OT (opened circle) and CP3MTOT (filled square), c P3HT (opened triangle), P3OT (opened circle) and CP3HT-OT (filled triangle) 

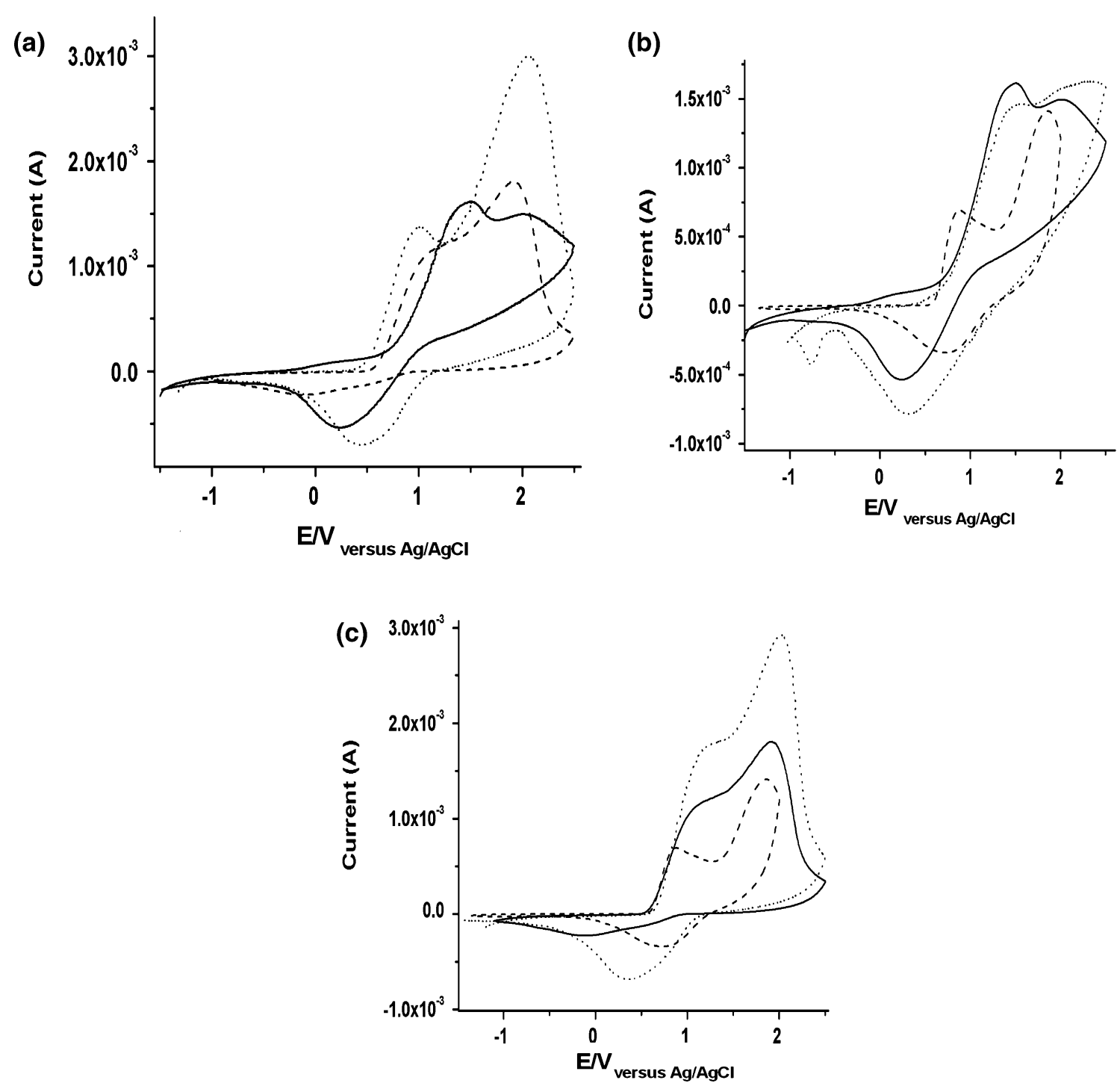

Fig. 4 Homo and copolymer cyclic voltammograms of $0.100 \mathrm{~mol} \mathrm{~L}^{-1} \mathrm{LiClO}_{4} / \mathrm{ACN}$, electrochemically synthesized on ITO. a P3MT (solid line), P3HT (dashed line) and CP3MT-HT (dotted

Figure 3 shows the Bode-phase diagrams made from the data generated through the EIS and OCP for the homo and copolymers on ITO. The Bode-phase diagram was represented to identify the influence of different processes for charge transfer in the conductivity of each system.

The results presented in the Bode-phase diagrams show that there was more than one phase at a different time constant for all systems, which was more common in the low frequencies to polaronic conduction and at high frequencies to electronic conduction [22]. This behavior was also seen in the deposition of P3ATs and copolymers between these and the PDFA on platinum electrode [18, 28]. The two time constants at low frequencies were related to the occurrence of the two-step process, referring to the line), b P3MT (solid line), P3OT (dashed line) and CP3MT-OT (dotted line), $\mathbf{c}$ P3HT (solid line), P3OT (dashed line) and CP3HT-OT (dotted line $) . \mathrm{v}=50 \mathrm{mV} \mathrm{s}^{-1}$

variations of radical cation (polaron) and dication (bipolaron) species in the polymer matrix.

In the Bode-phase diagrams for P3MT and P3OT homopolymers, Fig. 3, two stages were found at low frequencies in different time constants centered at 0.01 and $81 \mathrm{~Hz}$, and 0.01 and $161 \mathrm{~Hz}$, respectively. In the P3HT homopolymer diagram, an extended phase was found between 0.008 and $478 \mathrm{~Hz}$. The behavior of the phase at $0.01 \mathrm{~Hz}$ is possibly due to the polaronic conduction process, the result of stabilizing the radical cation, and the behavior of the phase in 81 and $161 \mathrm{~Hz}$ to bipolaronic conduction as a result of stabilizing the dication. Among $0.008-478 \mathrm{~Hz}$ must contain the stabilization for both radical cations and dications. 

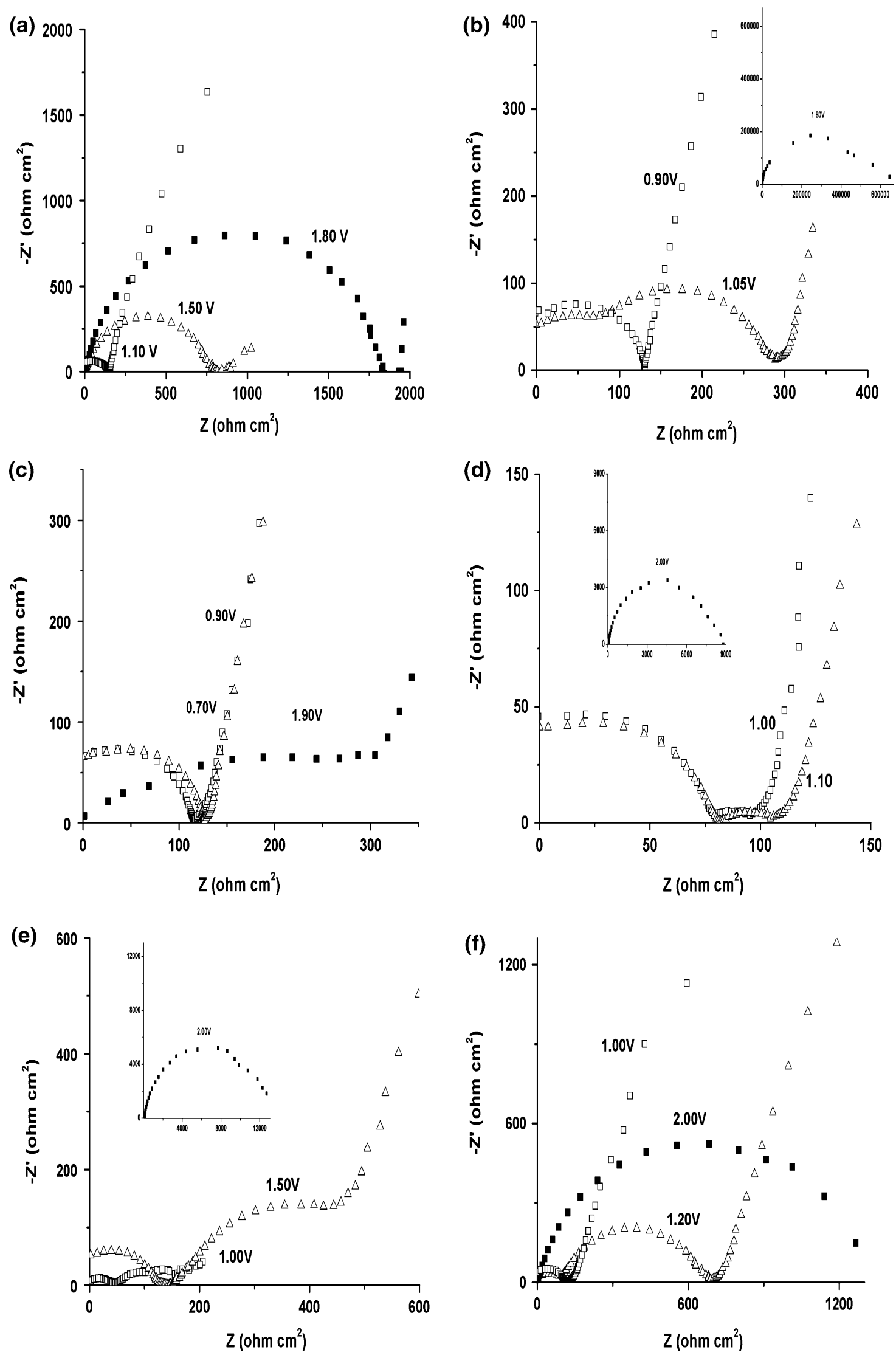
4Fig. 5 Nyquist diagrams obtained in different overpotentials for the following films deposited on ITO in $0.100 \mathrm{~mol} \mathrm{~L}^{-1}$ of $\mathrm{LiClO}_{4} / \mathrm{ACN}$ : a P3MT, b P3HT, c P3OT, d CP3MT-HT, e CP3MT-OT, f CP3HTOT

An increase in the angles of the phases in the following sequence $\mathrm{P} 3 \mathrm{OT}<\mathrm{P} 3 \mathrm{HT}<\mathrm{P} 3 \mathrm{MT}$ was also seen in the diagrams. This result indicates that there is greater oscillation on the P3MT film between the radical cation and dication in the polymer matrix. Thus, the variation between the angles of the phase of homopolymers confirm the results seen in the Nyquist diagrams, Fig. 1, wherein the P3MT showed a lower $\mathrm{R}_{\mathrm{CT}}$.

Two phases were also found in low frequencies centered on $0.24-18.20,1.00-37.92$ and $0.01-657.66 \mathrm{~Hz}$, respectively, in the Bode-phase diagrams for the CP3MT-HT, CP3MT-OT and CP3HT-OT copolymers. The largest angle in the phase was seen in the CP3MT-OT Bode diagram, Fig. 3b, and the smallest angle in the phase for CP3HT-OT. This data confirms the smaller $\mathrm{R}_{\mathrm{CT}}$ for CP3MT-OT among the copolymers given in the Nyquist diagram, Fig. 1b.

Figure 4 shows the cyclic voltammograms for the first potential scan started at $-1.50 \mathrm{~V}$ of the homo and copolymer films in $0.100 \mathrm{~mol} \mathrm{~L}^{-1}$ of $\mathrm{LiClO}_{4} / \mathrm{ACN}$ previously synthesized on ITO. Cyclic voltammograms recorded for all these films showed two more defined oxidation peaks than those taken from the films generated under similar conditions on the platinum electrode, which showed a wider oxidation peak. The cathodic behavior was similar to that seen on platinum $[26,28]$.

In the cyclic voltammograms, Fig. 4a, displacements of oxidation peaks and a reduction in the P3MT and P3HT homopolymers in relation to the CP3MT-HT copolymer was seen, with the oxidation displaced from $1.50-1.80 \mathrm{~V}$ and 1.05-1.80 to $1.00-2.00 \mathrm{~V}$, and for a reduction of 0.30 and $-0.09 \mathrm{~V}$ to $0.45 \mathrm{~V}$, respectively, demonstrating a different electrochemical behavior for the copolymer compared to their homopolymers. In Fig. 4b electrochemical behavior of the CP3MT-OT copolymer was similar to $\mathrm{P} 3 \mathrm{MT}$, i.e. the oxidation peaks occurred at 1.50-1.80 and $1.50-2.00 \mathrm{~V}$ and a reduction in 0.30 and $0.32 \mathrm{~V}$, respectively. With respect to the CP3MT-OT copolymer peaks, the potentials to oxidize and reduce P3OT are displaced, as they were seen at $0.90-1.90$ and $0.78 \mathrm{~V}$, respectively. In
Fig. 4c the electrochemical behavior of the CP3HT-OT copolymer was found to be different to the P3HT and P3OT homopolymers, displacing the potential for oxidation to $1.20-2.00 \mathrm{~V}$ and a reduction to $0.33 \mathrm{~V}$.

Considering the results obtained by cyclic voltammetry, the EIS technique was used in three overpotentials, these being determined before the first, and in the first and second oxidation peaks (Table 2), to monitor changes in the resistive behavior of the films, in both oxidation peaks that were seen for all systems studied.

Figure 5 shows the Nyquist diagrams obtained by EIS on the overpotential, which demonstrated the resistive behavior of homo and copolymers studied on ITO electrode.

For most of the Nyquist diagrams obtained on the different overpotentials, a semicircle was seen except in the copolymers and in some of the applied potentials. In such cases, a second semicircle appeared at higher frequencies, probably related to the characteristics of interfacial processes in terms of resistance in the ITO/polymer interface, characteristics not found in these copolymers on platinum $[3,23]$.

In the Nyquist diagrams for the homopolymers, when using the second overpotential, relating to the first oxidation peak, the increase in the diameter of the semicircle could be seen before the first peak. A significant increase was seen in diameter when the third potential was used on the second oxidation peak. Relating this result with the behavior seen in cyclic voltammetry of Fig. 4, the first oxidation peak may be related to the presence of a specific less resistant species than the species present in the second oxidation peak.

For the copolymers, the Nyquist diagrams shows that different behavior was seen for the homopolymers in the second potential. Only CP3MT-OT and CP3HT-OT saw an increase in the diameter of the semicircles in relation to them in the first potential; but for all copolymers applied to a third potential, the semicircle diameters increased in relation to them on the second potential, which may be related to the presence of a specific more resistive species when these films were submitted to a third potential.

Table 3 shows the values of charge transfer resistance $\left(\mathrm{R}_{\mathrm{CT}}\right)$ of the films generated on ITO, determined by the Nyquist diagrams, Fig. 5.
Table 3 Resistance of charge transfer $\left(\mathrm{R}_{\mathrm{CT}}\right)$ of the films obtained from the Nyquist diagrams

\begin{tabular}{lllllll}
\hline Oxidation potentials & \multicolumn{6}{l}{$\mathrm{R}_{\mathrm{CT}}\left(\Omega \mathrm{cm}^{2}\right)$} \\
\cline { 2 - 7 } & P3MT & P3HT & P3OT & CP3MT-HT & CP3MT-OT & CP3HT-OT \\
\hline Before the first peak & $1.50 \times 10^{2}$ & $1.18 \times 10^{2}$ & $1.13 \times 10^{2}$ & 99.8 & $1.53 \times 10^{2}$ & $1.01 \times 10^{2}$ \\
On first peak & $8.23 \times 10^{2}$ & $2.86 \times 10^{2}$ & $1.23 \times 10^{2}$ & $1.10 \times 10^{2}$ & $4.40 \times 10^{2}$ & $6.94 \times 10^{2}$ \\
On second peak & $1.83 \times 10^{3}$ & $6.31 \times 10^{5}$ & $3.03 \times 10^{2}$ & $8.78 \times 10^{3}$ & $1.25 \times 10^{4}$ & $1.26 \times 10^{3}$ \\
\hline
\end{tabular}



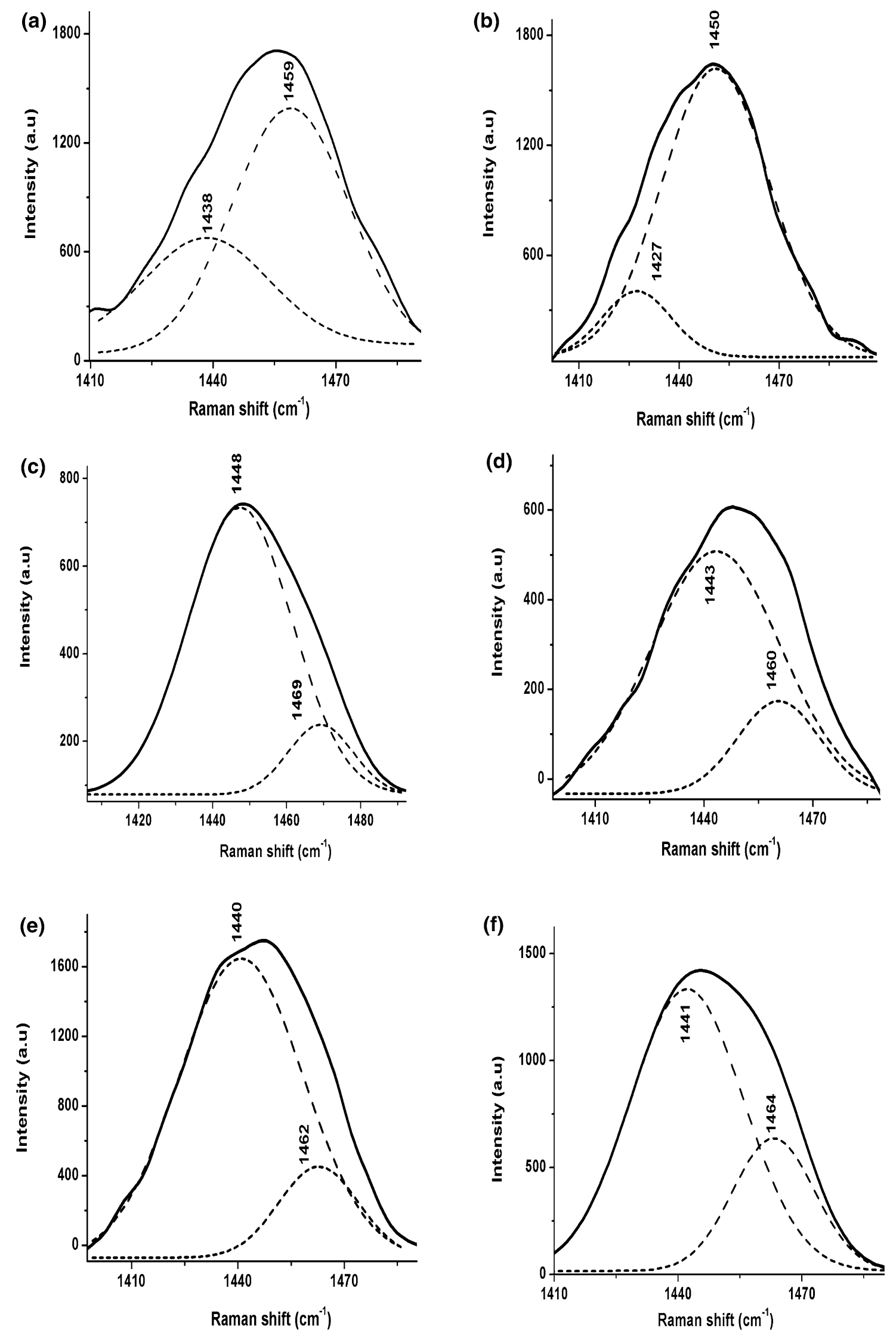
4Fig. 6 In situ Raman spectra deconvoluted by applying the indicated potential, obtained in $0.100 \mathrm{~mol} \mathrm{~L}^{-1}$ of $\mathrm{LiClO}_{4} / \mathrm{ACN}$ for the following films synthesized on ITO: a P3MT, b P3HT, c P3OT, d CP3MTHT, e CP3MT-OT, f CP3HT-OT

The $R_{C T}$ values described in Table 3 for the films on ITO confirm the increase in resistivity of these films, when using the potential of a second oxidation peak. Moreover, the decrease in resistivity of films on ITO of up to $10^{5} \Omega \mathrm{cm}^{2}$ could be found compared to $\mathrm{R}_{\mathrm{CT}}$ obtained in a more conductive strip (first potential) of films on platinum with $\mathrm{R}_{\mathrm{CT}}$ of $7.81 \times 10^{4}, \quad 7.91 \times 10^{4}, \quad 5.38 \times 10^{4}$, $9.43 \times 10^{3}, 3.52 \times 10^{3}$ and $2.28 \times 10^{7} \Omega \mathrm{cm}^{2}$ for P3MT, P3HT, P3OT, CP3MT-HT, CP3MT-OT and CP3HT-OT, respectively [3].

For a better understanding of the data seen by the IES technique in $\eta$, the in situ Raman spectra was obtained for the compounds studied at different potentials, aiming to identify the dication segments present in the polymeric material, proving a resistive character seen by increasing the anodic potential.

Figure 6 shows the deconvoluted in situ Raman spectra for homo and copolymers obtained by applying the potential near the second oxidation peak, as seen by cyclic voltammetry in $0.100 \mathrm{~mol} \mathrm{~L}^{-1}$ of $\mathrm{LiClO}_{4} / \mathrm{ACN}$, of the films generated on ITO. Less stable spectra was obtained for copolymers, first and second overpotential, which did not favor a deconvolution analysis by Raman spectra.

In the P3MT and P3HT deconvoluted in situ Raman spectra, Fig. 6a, b, when applying $1.80 \mathrm{~V}$, two bands with different relative intensities, frequencies set at 1,438 and $1,459 \mathrm{~cm}^{-1}, 1,427$ and $1,450 \mathrm{~cm}^{-1}$ were found with aromatic and dication characteristics, respectively, [6, 28, 29]. It was found that the bands were more intense in the dication species compared to the aromatic attribution, suggesting the most resistive nature of these two materials is due to the dication segments in the polymer matrix. In the P3OT deconvoluted in situ Raman spectra, Fig. 6c, when applying $1.90 \mathrm{~V}$, two defined bands were seen at frequencies of 1,448 and $1,469 \mathrm{~cm}^{-1}$, dication and radical cation characteristics, respectively, [6, 26-29], and that band referring to dication was more intense compared to the band attributed to the radical cation.

These results show that in the most anodic potentials, there was the most dication stabilization along the polymer matrices and even the presence of the radical cation segments in P3OT relating to the absence in the P3MT and $\mathrm{P} 3 \mathrm{HT}$, leaving the P3OT even more conductive than others (Table 3).

In the copolymer deconvoluted in situ Raman spectra, Fig. $6 \mathrm{~d}-\mathrm{f}$, two contributions were found with different relative intensities, defined in the frequencies between $1,440-1,443$ and $1,460-1,464 \mathrm{~cm}^{-1}$. In the CP3MT-HT spectra, Fig. 6d, by applying $1.60 \mathrm{~V}$, a $1,443 \mathrm{~cm}^{-1}$ band occurred, from the contribution of the P3HT homopolymer dication species, and a band at $1,460 \mathrm{~cm}^{-1}$ attributed to the dictation species from the P3MT, considering the resistive nature of the material generated. In the CP3MT-OT spectrum, Fig. 6e, dication bands were found at 1,440 and $1,462 \mathrm{~cm}^{-1}$ from the P3OT and P3MT, respectively. In the Raman spectra of these two copolymers, an intensified band was found in 1,443 and $1,440 \mathrm{~cm}^{-1}$ relating to the main diction contribution coming from the P3OT and $\mathrm{P} 3 \mathrm{HT}$, respectively, also considering the more resistive nature of the material generated. However, in the CP3HTOT spectrum, by applying $2.00 \mathrm{~V}$, two bands were found at 1,441 and $1,464 \mathrm{~cm}^{-1}$ frequencies, attributed to the dication species and radical cation of P3HT and P3OT respectively, with more intensity in the band at $1,441 \mathrm{~cm}^{-1}$, which is related to the stabilization of the dication in this copolymer matrix. The presence of the radical cation probably left $\mathrm{CP} 3 \mathrm{HT}-\mathrm{OT}$ more conductive than the others (Table 3).

In Fig. 7, the Bode-phase diagrams were presented for homo and copolymers simultaneously generated with $\mathrm{Ny}$ quist diagrams at different overpotentials.

In the P3MT Bode-phase diagram, Fig. 7a, at low frequencies, by applying a $1.10 \mathrm{~V}$ potential, a phase at $0.02 \mathrm{~Hz}$ was found relating to polaronic conduction, and at high frequencies, a phase around $1 \times 10^{5} \mathrm{~Hz}$ relating to electronic conduction [22]. After applying 1.50 and $1.80 \mathrm{~V}$ potentials, a decrease was found in the angle of the phase at $0.02 \mathrm{~Hz}$ and the appearance of long phases at 273.34 and $209.70 \mathrm{~Hz}$, relating to bipolaronic conduction. In these higher potentials, the angle of the phase at high frequencies decreased, showing that there was less electrical conduction in these cases. This behavior can be related to more resistivity of the polymer at higher overpotentials, as seen in the Nyquist diagrams, Fig. 5a. This result had been previously confirmed by deconvolution of the P3MT in situ Raman spectrum, Fig. 6a where this behavior is related to the stabilization of the dication species, responsible for bipolaronic conduction.

In the Bode-phase diagrams of P3HT and P3OT homopolymers, Fig. 7b, c, taken from 0.90 to 1.50 and 0.70 to $0.90 \mathrm{~V}$ overpotentials presented phases at 0.01 and $0.005 \mathrm{~Hz}$, respectively, at low frequencies, related to polaronic conduction. By applying 1.80 and $1.90 \mathrm{~V}$ potentials, a decrease in the angle of these phases was found and a phase centered on 19.50 and $43.86 \mathrm{~Hz}$ for P3HT and P3OT appeared, related to bipolaronic conduction. No variations in the angles of the phase occurred in these potentials and at high frequencies.

In Fig. 7d-f a decrease in the angle of the phase around $0.01 \mathrm{~Hz}$ is found for copolymers and phases appeared at 106.41, 248.40 and 90.70 Hz for CP3MT-HT, CP3MT-OT 
(a)
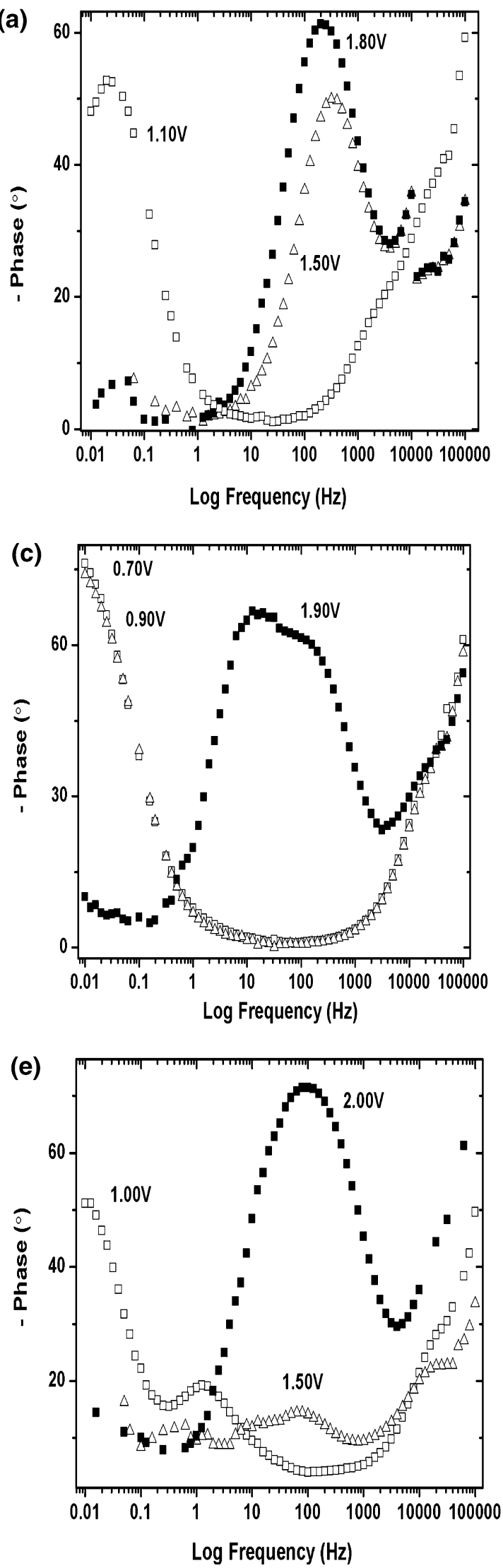
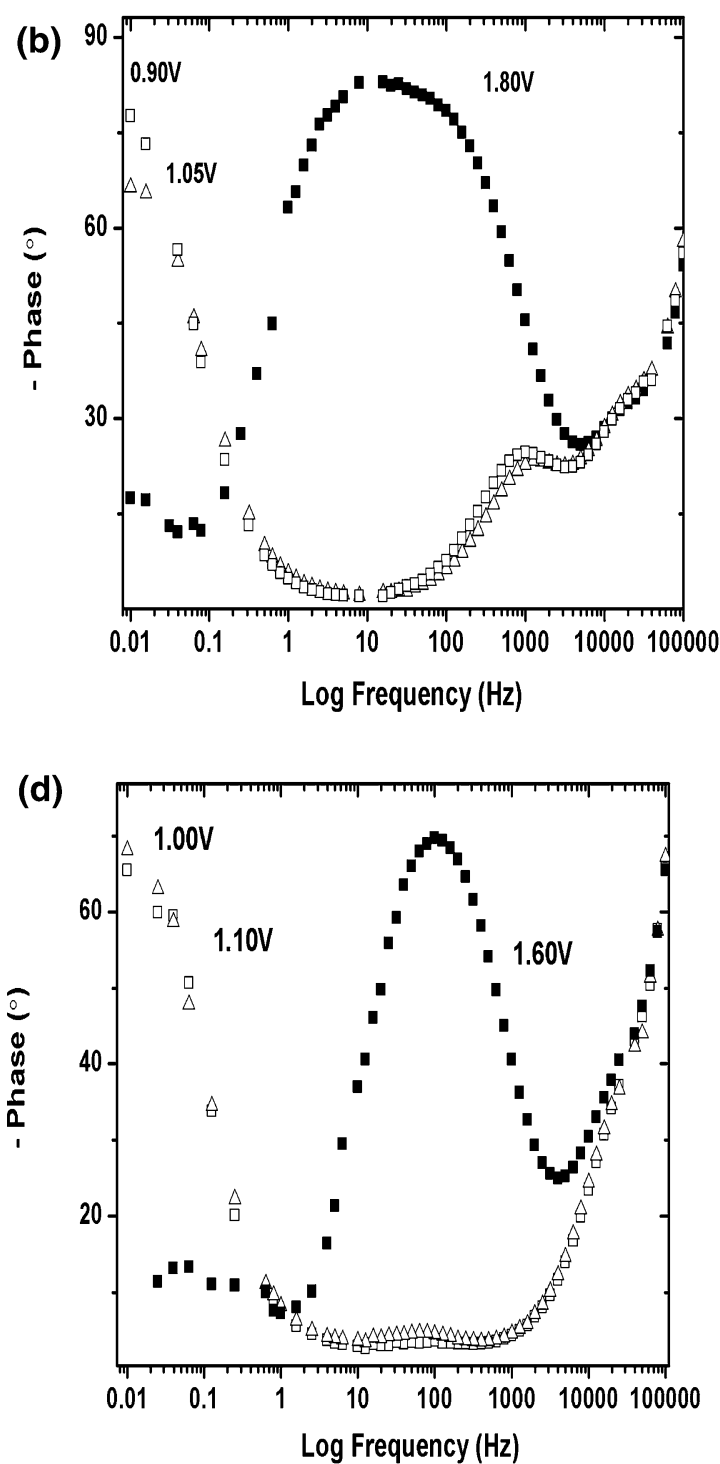

(f)

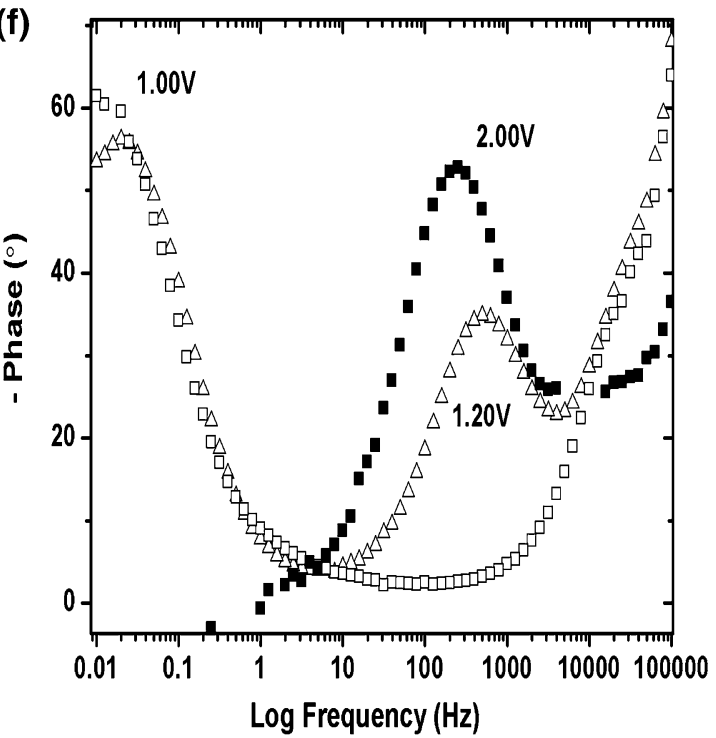


4Fig. 7 Bode-phase diagrams generated by EIS in different overpotentials for the following films deposited on ITO in $0.100 \mathrm{~mol} \mathrm{~L}^{-1}$ of $\mathrm{LiClO}_{4} / \mathrm{ACN}$ : a P3MT, b P3HT, $\mathbf{c}$ P3OT, d CP3MT-HT, e CP3MTOT, f CP3HT-OT

and CP3HT-OT films, respectively, by increasing overpotential.

High frequencies were not found in CP3MT-HT and CP3MT-OT, Fig. 7d, e, variations in the angle of the phase at different overpotentials, showing that the electronic conduction in this copolymer does not change at higher potentials. In the CP3HT-OT diagram, Fig. 7f, there was a decrease in angle of the phase with a $2.00 \mathrm{~V}$ potential, showing that this copolymer changed to electronic conduction at higher potentials.

\section{Conclusions}

The EIS data together with the characterization of homo and copolymers, electrochemically synthesized on ITO electrode, through the in situ and ex situ Raman spectra, confirm the presence of radical cation and dication segments in the polymer matrix.

Using the Nyquist diagrams, the $\mathrm{R}_{\mathrm{CT}}$ values show that, with a higher overpotential in the system studied, it resulted in an increase in charge transfer resistance. Supplementing these findings, we found more than one phase at different time constants using the Bode-phase diagrams, being able to relate the polaronic and bipolaronic conduction at low frequencies and to the electronic conduction at high frequencies.

Through in situ Raman spectra in the most anodic potentials, obtained by cyclic voltammetry, it was possible to prove the presence of more dication segments in the polymer matrix responsible for higher resistivity of materials, by the stabilization of the dication species in the polymer matrix.

Acknowledgments We would like to express our appreciation of the Spectroscopy Laboratory (SPEC) at the PROPPG/UEL Multiuser Center and the work was supported by CAPES PROGRAM/SPECIAL VISITING RESEARCHER (PVE), process No. 124/2012.

\section{References}

1. Z.W. Sun, A.J. Frank, J. Chem. Phys. 94, 4600 (1991)

2. S. Bertho, B. Campo, F. Piersimoni, D. Spoltore, J. D́Haen, L. Lutsen, W. Maes, D. Vanderzande, J. Manca, Sol. Energy Mater. Sol. Cells 110, 69 (2013)
3. T.N.M. Cervantes, D.C. Bento, E.C.R. Maia, R.V. Fernandes, E. Laureto, G.J. Moore, G. Louarn, H. de Santana, J. Mater. Sci. Mater. Electron. 25, 1703 (2014)

4. A. Tarola, D. Dini, E. Salatelli, F. Andreani, F. Decker, Electrochim. Acta 44, 4189 (1999)

5. M. Weis, K. Lee, D. Taguchi, T. Manaka, M. Iwamoto, Synth. Met. 162, 2236 (2012)

6. E.C.R. Maia, D.C. Bento, E. Laureto, D.A.M. Zaia, E.M. Therézio, G.J. Moore, H. de Santana, J. Serb. Chem. Soc. 78, 507 (2013)

7. D. Kaduwal, B. Zimmermann, U. Würfel, Sol. Energy Mater. Sol. Cells 124, 449 (2014)

8. A. Bagui, S.S.K. Iyer, Org. Electron. 15, 1387 (2014)

9. Y.-J. Cheng, S.-H. Yang, C.-S. Hsu, Chem. Rev. 109, 5868 (2009)

10. S. Ben Dkhil, R. Ebdelli, W. Dachraoui, H. Faltakh, R. Bourguiga, J. Davenas, Synth. Met. 192, 74 (2014)

11. D.R. Rosseinsky, R.J. Mortimer, Adv. Mater. (Weinheim, Ger.) 13, 783 (2001)

12. D. Kaduwal, H.-F. Schleiermacher, J. Schulz-Gericke, T. Kroyer, B. Zimmermann, U. Würfel, Sol. Energy Mater. Sol. Cells 124, 92 (2014)

13. L.-M. Chen, Z. Hong, G. Li, Y. Yang, Adv. Mater. (Weinheim, Ger.) 21, 1434 (2009)

14. J. Mikroyannidi, A.N. Kabanakis, S.S. Sharma, G.D. Sharma, Adv. Funct. Mater. 21, 746 (2011)

15. J. Xiao, W. Chen, F. Wang, J. Du, Macromolecules (Washington, DC, U. S.) 46, 375 (2013)

16. S. Wilken, D. Scheunemann, V. Wilkens, J. Parisi, H. Borchert, Org. Electron. 13, 2386 (2012)

17. A. Bou, P. Torchio, S. Vedraine, D. Barakel, B. Lucas, J.-C. Bernède, P.-Y. Thoulon, M. Ricci, Sol. Energy Mater. Sol. Cells 125, 310 (2014)

18. D.C. Bento, E.C.R. Maia, P.R.P. Rodrigues, G. Louarn, H. de Santana, J. Mater. Sci. Mater. Electron. 24, 4732 (2013)

19. E.G. Tolstopyatova, S.N. Sazonova, V.V. Kondrat'ev, V.V. Malev, Russ. J. Electrochem. 40, 930 (2004)

20. M. Ates, T. Karazehir, F. Arican, N. Eren, J. Coat. Technol. Res. 10, 317 (2013)

21. H. Ding, L. Pigani, R. Seeberb, C. Zanardi, J. New Mater. Electrochem. Syst. 3, 339 (2000)

22. G. Lillie, P. Payne, P. Vadgama, Sens. Actuators B 78, 249 (2001)

23. L.F.Q.P. Marchesi, F.R. Simões, L. Pocrifka, E.C. Pereira, J. Phys. Chem. B 115, 9570 (2011)

24. H. Ding, Z. Pan, L. Pigani, R. Seeber, C. Zanardi, Electrochim. Acta 46, 2721 (2001)

25. E.G. Tolstopyatova, S.N. Sazonova, V.V. Malev, V.V. Kondratiev, Electrochim. Acta 50, 1565 (2005)

26. D.C. Bento, E.C.R. Maia, T.N.M. Cervantes, R.V. Fernandes, E. Di Mauro, E. Laureto, M.A.T. da Silva, J.L. Duarte, I.F.L. Dias, H. de Santana, Synth. Met. 162, 2433 (2012)

27. T.N.M. Cervantes, D.C. Bento, E.C.R. Maia, D.A.M. Zaia, E. Laureto, M.A.T. Silva, G.J. Moore, H. de Santana, J. Mater. Sci. Mater. Electron. 23, 1916 (2012)

28. H. De Santana, E.C.R. Maia, D.C. Bento, T.N.M. Cervantes, G.J. Moore, J. Mater. Sci. Mater. Electron. 24, 3352 (2013)

29. W.J. Doherty, N.R. Armstrong, S.S. Saavedra, Chem. Mater. 17, 3652 (2005) 